\title{
The Principles and Laws of Black Hole Universe
}

\author{
T. X. Zhang \\ Department of Physics, Alabama A \& M University, Normal, AL, USA \\ Email: tianxi.zhang@aamu.edu
}

How to cite this paper: Zhang, T.X. (2018) The Principles and Laws of Black Hole Universe. Journal of Modern Physics, 9, 1838-1865.

https://doi.org/10.4236/jmp.2018.99117

Received: July 15, 2018

Accepted: August 14, 2018

Published: August 17, 2018

Copyright $\odot 2018$ by author and Scientific Research Publishing Inc. This work is licensed under the Creative Commons Attribution International License (CC BY 4.0).

http://creativecommons.org/licenses/by/4.0/

\begin{abstract}
A new alternative cosmological model called black hole universe was recently developed by the author on the basis of the following three fundamentals: 1) the principle of spacetime black hole equivalence, 2) the cosmological principle of spacetime isotropy and homogeneity, and 3) the Einsteinian general theory of relativity that describes the effect of matter on spacetime. According to this black hole model of the universe, the author up-to-dately has self-consistently described the origin, structure, evolution, expansion, and acceleration of the universe, quantitatively explained the measurements of cosmic microwave background radiation, type Ia supernovae's luminosity distance and redshift, and dynamic properties of star-like, massive, and supermassive black holes such as gamma-ray bursts, X-rays flares from galactic centers, and quasars, and fully overcome the difficulties of the conventional model of the universe such as the problems of horizon, flatness, monopole, inflation, dark matter, dark energy, and so on. In this paper, the author will examine and overview thoroughly this new cosmological model and completely describe its development from the three fundamentals and its creative explanations to the existing observations of the universe. From this comprehensive investigation of the new cosmological model, the author will further reveal the fundamental regularities and laws of the black hole universe with respect to the spacetime mass and radius, spacetime equilibrium, spacetime expansion and acceleration, spacetime radiation energy, and spacetime entropy variation. These efforts will help us to uncover various regularities and mysteries of the universe.
\end{abstract}

\section{Keywords}

Cosmology, Black Hole, Spacetime, Universe

\section{Introduction}

Brewing the idea-the universe as a black hole-that was unexpectedly emerged 
from his mind in 2004 at reading the famous paper entitled "Mach's principle and a relativistic theory of gravitation [1]" in the library of the University of Alabama in Huntsville to develop his new electric redshift mechanism [2] and his new gravitational shielding theory [3] for three years, Dr. T. X. Zhang formally proposed a new cosmological model called black hole universe in the $211^{\text {th }}$ American Astronomical Society (AAS) meeting held at Austin, Texas (USA), on January 7-11, 2008 [4]. This new multiverse cosmological model is consistent with Mach's principle, governed by Einstein's general theory of relativity, and able to explain all the existing observations of the universe according to the well-developed physics without encountering any fates of the universe or difficulties [5]. Seven years later, Zhang [6] further successfully convened and chaired a special session entitled "Observation and Theory for Multiverse" for the 224 $4^{\text {th }}$ AAS meeting held at Boston, Massachusetts (USA), on June 1-5, 2014. In this special session of the $224^{\text {th }}$ AAS meeting, many famous cosmologists from the world were brought together to discuss cosmological issues of multiverse [7]. Zhang [8] also gave a talk on his hierarchically multilayered black hole universe model and greatly impacted the communities of astronomy, astrophysics, and cosmology with this new cosmological model.

The early studies done by the author up to the date have 1) self-consistently described the origin of the universe from black holes, the structure of the entire space with infinite layers, the iterative and cyclic evolution in an endless and beginningless style, and the physical expansion outward in one-way along the time or entropy increasing direction, 2) quantitatively explained the measurement of $2.725 \mathrm{~K}$ cosmic microwave background radiation with the blackbody spectrum, the observation of type Ia supernovae's luminosity distance and redshift for the acceleration of the universe, and the dynamic properties of star-like, massive, and supermassive black holes such as gamma-ray bursts, X-rays flares from galactic centers, and quasars, and 3) fully overcome the difficulties of the conventional standard big bang model of the universe such as the problems of the horizon, flatness, monopole, inflation, dark matter, dark energy, and so on. The results obtained from these early studies have been presented in a sequence of AAS meetings [4] [6]-[17] and published in a number of refereed journals and conference proceedings [5] [18]-[25].

The big bang model of the universe, though being the currently accepted standard cosmological model, critically relies on a growing number of hypothetical entities [26]. Without making these supplementary assumptions, the big bang theory severely contradicts with the observations of the universe. Theoretically, the big bang universe stands only on the basis of two fundamentals, which are the cosmological principle of spacetime isotropy and homogeneity and Einstein's general theory of relativity, but needs to face lots of uncertainties and difficulties that are emerged in explaining the observations of the universe ([13] [25], see also left panel of Figure 1). To overcome these difficulties, cosmologists had to make a number of supplementary assumptions. For instances, the inflation epoch was assumed in order for the big bang model to solve the initial value 


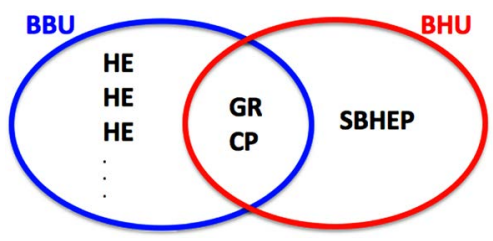

Figure 1. The big bang universe (BBU) vs. the black hole universe (BHU) in fundamentals [13] [25]. BBU was developed on the basis of Einstein's general relativity (GR) and the cosmological principle (CP) with innumerable hypothetical entities (HEs) to explain observations and overcome cosmic problems and difficulties. BHU was developed on the basis of GR, CP and the principle of spactime black hole equivalence (SBHEP). With this new principle (i.e. SBHEP), BHU can also explain all the existing observations of the universe and meantime overcome the cosmic problems and difficulties in terms of the well-developed physics without needing any other HEs.

problems such as the horizon, flatness, and monopole problems [27]. The dark energy component is introduced in order for the big bang theory to address the acceleration of the universe as shown by the measurement of type Ia supernovae's redshift and luminosity distance [28] [29]. These hypothetical entities are growing in number as we explore the universe more deeply and more completely.

In the black hole model of the universe [4] [5], Zhang proposed a new postulate or principle: the Principle of Spacetime Black Hole Equivalence (SBHEP), in addition to the cosmological principle of spacetime isotropy and homogeneity and Einstein's general theory of relativity [13] [25]. By upstanding on these three fundamentals, this new black hole universe model not only self-consistently explains all the existing observations of the universe, but also naturally overcomes the difficulties of the universe without requiring any extra hypotheses (see the right panel of Figure 1). Therefore, one principle can remove all hypothetical entities from the big bang model of the universe. In this paper, we will thoroughly examine this new cosmological model and fully describes its development from the three fundamentals and its explanations to the existing observations of the universe. From this complete investigation of the new cosmological model, we will further reveal the fundamental regularities and laws of the black hole universe with respect to the spacetime mass and radius, spacetime equilibrium, spacetime expansion and acceleration, spacetime radiation conservation, and spacetime entropy variation. These efforts will help us to develop an elegant, perfect, and complete cosmological model that reveals various aspects and mysteries of the universe.

\section{The Principles of Black Hole Universe}

\subsection{The Principle of Spacetime Black Hole Equivalence}

The first principle or fundamental of black hole universe is the principle of spacetime black hole equivalence. More specifically, a black hole constructs an individual spacetime and a spacetime encloses a black hole. Black holes and spacetimes are equivalent (Figure 2). This principle comes from the fact or 


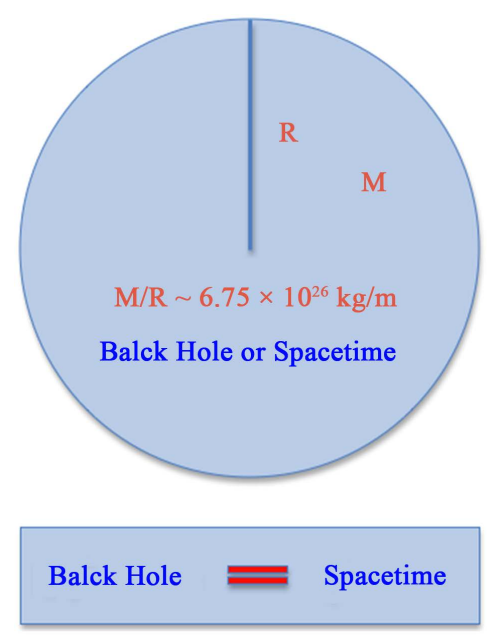

Figure 2. Equivalence between spacetime and black hole. A region, where matter accumulates up to a critical point such that $M / R \sim 6.75 \times 10^{26} \mathrm{~kg} / \mathrm{m}$, forms a black hole and meantime constructs its own space to be singular to the outside and its own time to be noncausal to the outside. Black holes and spacetimes are equivalent. This principle of spacetime black hole equavalence is one of the three fundamentals of the black hole universe model.

result of Einstein's general theory of relativity and Schwarzschild's solution of the field that a region, where matter accumulates up to a critical point such that the mass-radius ratio is equal to $M / R=c^{2} / 2 G \sim 6.75 \sim 10^{26} \mathrm{~kg} / \mathrm{m}$ (or $\sim 0.3375$ solar masses $/ \mathrm{km}$ ), forms a black hole and meantime builds or constructs its own space being singular to the outside and its own time being noncausal to the outside. When a black hole is formed, an individual spacetime is immediately wrapped. Here $c$ is the speed of light traveling in the free space and $G$ is the gravitational constant.

According to this newly proposed principle, our four-dimensional (4D) spacetime universe is a black hole, extremely supermassive and fully expanded. All the inside, currently observed, star-like, massive, and/or supermassive black holes are subspacetimes of our black hole universe (Figure 3). The bottom panel of Figure 3 represents our universe spacetime as a line, which breaks with or contains a number of holes as subspacetimes. Here, we, for simple, only drew four subspacetimes, corresponding to four star-like or supermassive black holes.

The spatial boundary of a spacetime or black hole including our black hole universe is the Schwarzschild absolute event horizon determined by [30]

$$
\frac{2 G M}{c^{2} R}=1
$$

where $M$ and $R$ are the mass and radius of the spacetime or black hole. This mass-radius relation is also the relation of the effective mass and radius of the universe from the Mach principle [31] [32] or the relation of the observable mass and radius of the universe. For convenience, here we name this relation as the Mach-Schwarzschild mass-radius (M-R) relation or as the Mach-Schwarzschild law of spacetime mass and radius, or the $0^{\text {th }}$ law of the black hole universe. 


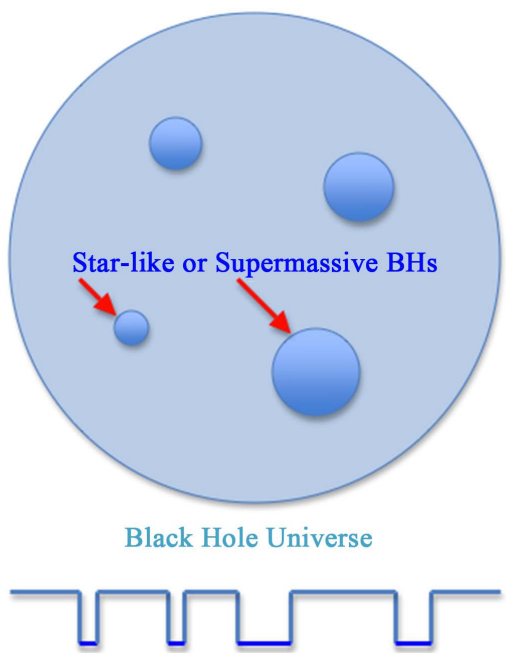

Figure 3. Our spacetime universe is an extremely supermassive and fully expanded black hole. The inside star-like, massve, and/or supermassive black holes observed are subspacetimes of our black hole universe.

Therefore, the black hole model of the universe does not exist the horizon problem. Within a black hole or a spacetime, an event occurred at one point is observable to all other points and events occurred at different points are all causally related one another.

Equation (1) indicates that the radius of a spacetime or black hole including the black hole universe is proportional to its mass $(R \propto M)$, i.e. the ratio remains a constant or is conserved. For a star-like black hole with 3 solar masses, its radius is about $9 \mathrm{~km}$. For a supermassive black hole with 3 billion solar masses, its radius is about $9 \times 10^{9} \mathrm{~km}$. For the present black hole universe with hundred billion trillion solar masses, its radius is about $10^{23} \mathrm{~km}$. The black hole model of the universe is supported by the Mach principle, the observations of the universe, and the Einsteinian general theory of relativity.

A black hole curves the spacetime maximally, so that it becomes singular and noncausal to the outside. It is highly curved, wrapped and closed. The 3D space curvature constant of a closed spacetime or a black hole including our black hole universe is

$$
k=1
$$

Therefore, the black hole model of the universe does not exist the flatness problem. Having neither the horizon problem nor the flatness problem, the black hole model of the universe does not need an inflation epoch, a nonphysical process that the current widely accepted big bang model of the universe is relying on [27], at all. Most inflation models predict the gravitational waves to be stronger and stronger, but observations showed opposite results [33] [34].

It is reasonable to suggest that our black hole universe was originated from a star-like black hole with several solar masses, which was formed from a massive star when the inside thermonuclear fusion has completed, and grew up through a supermassive black hole with billions of solar masses to the present state with 
hundred-billion-trillions of solar masses, by accreting the ambient matter or merging with other black holes (Figure 4). It is not important which black hole is the first one to form the present universe because its contribution is waned out and negligible. This is analogy to that a big river is originated or formed by merging a great number of streams, but which stream is the first one is not essential. The black hole model of the universe is not sensitive to its past (no initial value or fine-tuning problem) or to the properties of star-like or supermassive black holes such as their angular momenta, temperatures, and sizes. The recent detections of gravitational waves by the Laser Interferometer Gravitational-Wave Observatory (LIGO) have confirmed the existence of stellar black holes and their mergers in the universe [35].

\subsection{The Cosmological Principle of Spacetime Isotropy and Homogeneity}

The second principle or fundamental of black hole universe is the cosmological principle of spacetime isotropy and homogeneity. This is also one of the two fundamentals in the big bang theory. According to this principle, a spacetime or black hole including our black hole universe, if it is viewed on a scale that is sufficient large or comparable to the scale of the spacetime, is homogeneous and isotropic. This principle implies that there is no special location and direction in a spacetime or black hole including the black hole universe. The properties of the universe are the same for all observers in the universe. More strongly, physical laws are all universal. If a physical law is applicable to the earth, then it can be applied to everywhere.

The metric of an isotropic and homogeneous spacetime or black hole including our black hole universe is given by the Friedmann-Lemaitre-Robertson-Walker (FLRW) metric [36] [37] [38] [39].

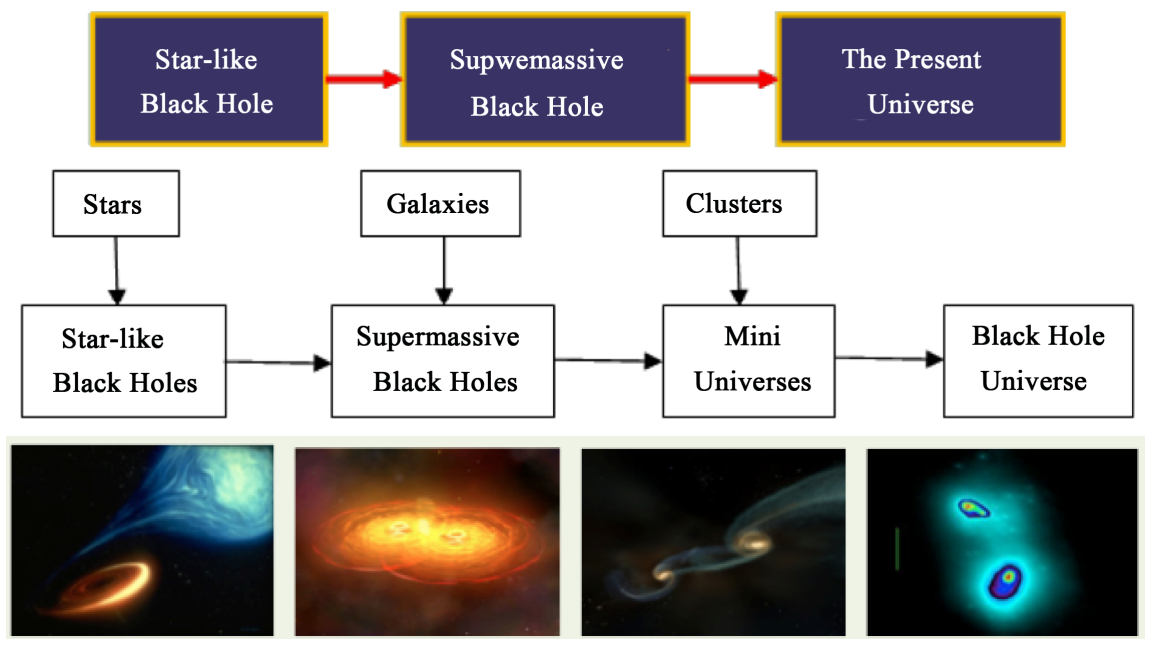

Figure 4. Our present black hole universe was originated from a star-like black hole with several solar masses, which grew through a supermassive black hole with billions of solar masses and further to the present state with hudred-billion-trillions of solar masses by accreting its ambient matter and merging with other black holes. 


$$
\mathrm{d} s^{2}=c^{2} \mathrm{~d} t^{2}-R^{2}(t)\left[\frac{\mathrm{d} r^{2}}{1-k r^{2}}+r^{2}\left(\mathrm{~d} \theta^{2}+\sin ^{2} \theta \mathrm{d} \phi^{2}\right)\right]
$$

where $d s$ is the line element of $4 \mathrm{D}$ spacetime, $r, \theta, \phi$ are co-moving spacecoordinates, $t$ is the time, $R(t)$ is the radius of curvature of the space, and $k$ is the curvature constant of the space. In the black hole model of the universe, $R(t)$ is the radius of the black hole or spacetime and $k=1$.

The density of matter ( $\rho$, defined as mass $M$ divided by the volume $V$ ) in an isotropic and homogeneous spacetime or black hole including our black hole universe can be determined as

$$
\rho \equiv \frac{M}{V}=\frac{3 c^{6}}{32 \pi G^{3} M^{2}}=\frac{3 c^{2}}{8 \pi G R^{2}}
$$

i.e. $\rho R^{2}=$ constant and $\rho M^{2}=$ constant . Here, we have applied the MachSchwarzschild M-R relation (Equation (1)) and $V=4 \pi R^{3} / 3$. It is seen that the density of matter in a spacetime is inversely proportional to the square of the mass ( $\rho \propto M^{-2}$ ) or the square of the radius $\left(\rho \propto R^{-2}\right.$ ). Both the mass and radius of the universe can be determined from the density of the universe. According to the current observations of the universe, the density of the present universe $\rho_{0}$ is about the critical density $\rho_{0} \sim \rho_{C} \equiv 3 H_{0}^{2} /(8 \pi G) \sim 9.2 \times 10^{-27} \mathrm{~kg} / \mathrm{m}^{3}$. Then, from Equation (4), we can determine the radius and mass of the present universe as $R_{0}=\sqrt{3 c^{2} /\left(8 \pi G \rho_{0}\right)} \sim 1.32 \times 10^{26} \mathrm{~m} \quad$ (or $\sim 14$ billion light years) and $M_{0}=R_{0} c^{2} /(2 G) \sim 8.8 \times 10^{52} \mathrm{~kg}$, respectively. Figure 5 plots the density of a black hole (solid line) as a function of the radius (or mass by $1 \mathrm{~km}=0.3375$ solar masses). The dotted line refers to the density of the black hole universe at the present time $\rho_{0}$. Its intersection with the solid line determines the radius and mass of the present black hole universe. The big bang model is not able to know

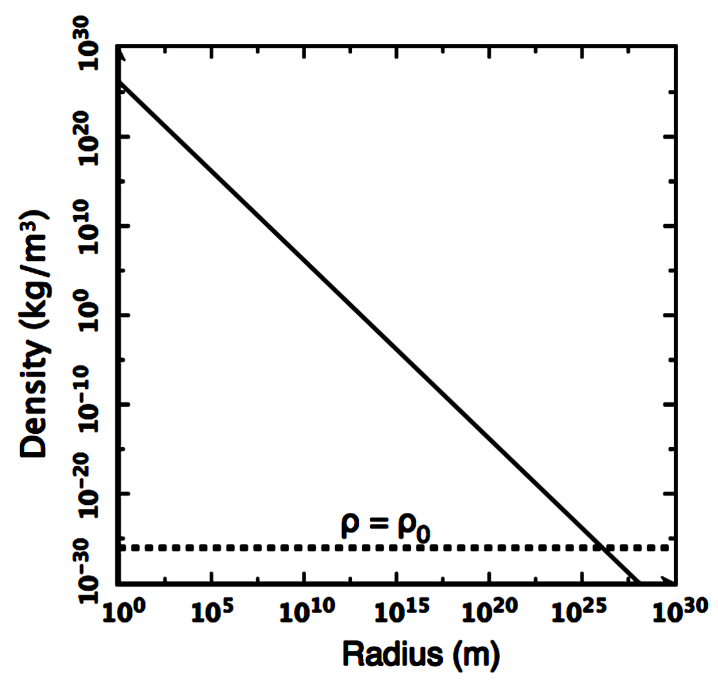

Figure 5. The density of a spacetime or black hole including our black hole universe versus the radius. The dotted line refers to $\rho=\rho_{0}$, so that its intersection with the solid line determines the radius and thus mass of the present black hole universe $R_{0}$ and $M_{0}$ to be in the orders of $\sim 10^{26} \mathrm{~m}$ and $\sim 10^{53} \mathrm{~kg}$, respectibvely. 
the mass of the universe and thus cannot determine the radius of the present universe according to the measurement of the density. Equation (4) can be considered as another form of the $0^{\text {th }}$ law of black hole universe.

\section{The Theory of Black Hole Universe}

\subsection{The Theory of Spacetime}

The theory or third fundamental of black hole universe is the Einsteinian general theory of relativity, which is a theory of spacetime or a geometric theory of gravitation that describes the effect of matter on spacetime [40]. The direct relation of the curvature of spacetime to the energy and momentum of matter in the spacetime is given by the Einsteinian field equation,

$$
G_{\mu v}=\frac{8 \pi G}{c^{4}} T_{\mu v}
$$

where $G_{\mu v}$ is the Einsteinian curvature tensor of spacetime and $\underline{T}_{\underline{\mu}}$ is the energy-momentum tensor of matter in spacetime. Einstein's another theory called special relativity describes the effect of motion on spacetime [41].

The theoretical predictions of general relativity such as the gravitational redshift of light from the Sun, the deflection of starlight by the Sun, the precessions of planetary perihelia, and the time delays of radar echoes have been confirmed in all observations and experiments to date. The Schwarzschild metric solution of the Einsteinian field equation of general relativity implies the existence of black holes, regions of space in which space and time are distorted in such a way that nothing, not even light, can escape from [30]. The observed gamma-ray bursts, X-rays flares from galactic centers, and quasars are believed to result from the activities of dynamic star-like, massive, and supermassive black holes, respectively. Not event light, except for the Hawking radiation [42], being able to escape from a black hole is only true if the black hole is in static state, in which the black hole has an enclosed event horizon. It should be noted that the Hawking radiation is a quantum effect obtained when the quantum mechanics (rather than a quantum gravity which has not yet been developed) is inappropriately applied to the surface of a black hole. As pointed out by Zhang in [18], a black hole, if it accretes its ambient matter or merges with other black holes, becomes dynamic. The event horizon of a dynamic black hole is temporarily broken and inside hot blackbody radiation leaks out and produces a gamma ray burst, $\mathrm{X}$-ray flare, or quasar if the black hole is a star-like, massive, or supermassive black hole, respectively [12] [16] [19] [22] [23] [24].

\subsection{The Theory of Cosmology}

Substituting the FLRW metric of spacetime (Equation (3)) into the Einsteinian field equation of general relativity (Equation (5)), we have the Friedmann equation [43],

$$
H^{2}(t) \equiv \frac{\dot{R}^{2}(t)}{R^{2}(t)}=\frac{8 \pi G \rho(t)}{3}-\frac{c^{2}}{R^{2}(t)}
$$


where $H(t)$ is the Hubble parameter or the expansion rate of the universe. The dot sign refers to the derivative with respect to time, $\dot{R}(t) \equiv \mathrm{d} R(t) / \mathrm{d} t$. The Hubble constant $H_{0}$ is the expansion rate of the universe at the present time and was measured as $H_{0} \sim 70 \mathrm{~km} / \mathrm{s} / \mathrm{Mpc}$ [44] [45] [46]. In addition, to derive Equation (6), we have considered Equation (2) for $k=1$. All spacetimes are governed by the Friedmann equation that is derived from the Einsteinian general theory of relativity with the FLRW metric, the Mach-Schwarzschild M-R relation, and the positive space curvature.

\section{The Laws of Black Hole Universe}

\subsection{The Law of Spacetime Equilibrium}

Substituting the density equation (Equation (4)) into the Friedmann equation (Equation (6)), we have

$$
\dot{R}(t)=0 \text { or } H(t)=0
$$

This implies that an individual spacetime or black hole including our black hole universe, if there is not any external influence, is in the static state and equilibrium. A black hole in the static or equilibrium state is quite or does not emit. We here call this regularity as the law of spacetime equilibrium (or the $1^{\text {st }}$ law of black hole universe). To have a static universe, Einstein [47] introduced a cosmological constant $\Lambda$ into the field equation (Equation (5)) of his general relativity. In the black hole model of the universe, we do not need a cosmological constant in order for the universe to be static. A spacetime or black hole including our black hole universe remains in the static state until an external influence such as matter entering is applied. Any internal event may also disturb or ripple the spacetime [35] [48]. A static spacetime does not expand and shrink due to equilibrium. In the spacetime equilibrium, the black hole remains its radius $R(t)$ or mass $M(t)$ as a constant, i.e. independent of time.

This law of spacetime equilibrium has overturned the traditional idea that all the matter inside a black hole is piled up in a singular point at the center, while other parts are empty, though nobody understands how this central singularity works. According to this law, the matter inside a black hole is homogeneously and isotropically distributed in the spacetime that the black hole forms. The spacetime formed by a black hole is highly curved and power enough to hold or sustain exactly all the matter of the black hole that forms the spacetime. The gravity of the matter inside a black hole is balanced by the curvature of the spacetime formed by the black hole. This does not exclude any possible anisotropy and inhomogeneity in a small scale, a scale that is significantly smaller than the scale of the spacetime formed by the black hole. The balance force produced by a highly curved spacetime (here we name it as the spacetime tension) prevents the matter of black hole from further crunching to the center to form a singular point. This is analogy to that a spring or string, when stretched by an object that is hung on, produces a tension that prevents the object from falling to the ground. 


\subsection{The Law of Spacetime Expansion}

When a spacetime or black hole including our black hole universe accretes matter from the outside or merges with other black holes, it becomes dynamic and expands [4] [5]. Considering that a spacetime or black hole including our black hole universe with mass $M(t)$ and radius $R(t)$ accretes matter of amount $\mathrm{d} M(t)$ from the outside, we have

$$
\frac{2 G(M(t)+\mathrm{d} M(t))}{c^{2} R(t)}>1
$$

In this case, the spacetime breaks its equilibrium state and expands its size or radius from $R(t)$ to $R(t)+\mathrm{d} R(t)$, where the radius increment $\mathrm{d} R(t)$ can be determined by

$$
\frac{2 G(M(t)+\mathrm{d} M(t))}{c^{2}(R(t)+\mathrm{d} R(t))}=1
$$

Substituting the Mach-Schwarzschild M-R relation Equation (1) into Equation (9), we have

$$
\frac{2 G}{c^{2}} \frac{\mathrm{d} M(t)}{\mathrm{d} R(t)}=1
$$

Therefore, a spacetime or black hole including our black hole universe expands when it inhales matter from the outside. The expansion rate (or the rate of change in radius) is obtained as

$$
\dot{R}(t)=\frac{2 G}{c^{2}} \dot{M}(t)
$$

and the Hubble parameter is given by

$$
H(t) \equiv \frac{\dot{R}(t)}{R(t)}=\frac{\dot{M}(t)}{M(t)}
$$

Equation (11) or Equation (12) indicates that the rate, at which a spacetime or black hole including the black hole universe expands, is proportional to the rate, at which it inhales matter from the outside. We here define this relation as the law of spacetime expansion, $\dot{M}(t)=H(t) M(t)$ or $\dot{R}(t)=H(t) R(t)$. A spacetime or black hole becomes dynamic when it accretes matter. A dynamic spacetime expands due to non-equilibrium.

Considering that a black hole with three solar masses accretes matter at a rate of $10^{-5}$ solar masses per year from its outside [49], we have $\dot{R}(t)=10 \mathrm{~cm} /$ years and $H(t) \sim 10^{7} \mathrm{~km} / \mathrm{s} / \mathrm{Mpc}$. Considering that a supermassive black hole with one billion solar masses swallows matter at a rate of one thousand solar masses in one year to run a quasar, we have $\dot{R}(t) \sim 3 \times 10^{3} \mathrm{~km} /$ years and $H(t) \sim 10^{6}$ $\mathrm{km} / \mathrm{s} / \mathrm{Mpc}$. When the black hole merges with other black holes, the growth rate should vary and be much larger in average than that when the black hole accretes its ambient matter. For our universe at the present state, the value of the Hubble parameter is measured as $H\left(t_{0}\right) \sim 70 \mathrm{~km} / \mathrm{s} / \mathrm{Mpc}$ [44] [45] [46]. If the 
radius of the universe is chosen as 13.7 billion light years, we have $\dot{R}\left(t_{0}\right) \sim c$, which implies that our universe is expanding in about the light speed at present. To have such fast expansion, the universe must inhale $\dot{M}\left(t_{0}\right)=\left[c^{2} /(2 G)\right] \dot{R}\left(t_{0}\right) \sim c^{3} /(2 G) \sim 2 \times 10^{35} \mathrm{~kg} / \mathrm{s}$, about $10^{5}$ solar masses in one second, or swallows a supermassive black hole in about a few hours. If $\Lambda$ is introduced to the Einsteinian field equation, the black hole universe model determines it as $\Lambda=3 H_{0}^{2}=3\left(\dot{M}_{0} / M_{0}\right)^{2} \sim 1.5 \times 10^{-35} \mathrm{~s}^{-2}$.

To expand, a spacetime or black hole including our black hole universe must have an outside, where matter is available for accretion and other black holes or parallel universes for mergers. Therefore, the black hole model of the universe suggests that the entire space is structured with layers, hierarchically and family-likely built [4] [5]. To see the hierarchical structure of the space, we sketch, in Figure 6, the innermost three layers of the black hole universe including the mother or parent universe, our universe itself, and child universes (i.e. stellar or supermassive black holes). For the sketch to be simple, we have only drawn three child universes and did not draw sister universes, which are universes parallel to our black hole universe. There should have a number of child universes and may also have many sister universes. A child universe grows by accreting materials from this universe or by merging with other child universes. This universe grows up by accreting materials from the mother universe or by merging with sister universes. The mother universe will also grow up if it has an outside. Otherwise, it is static.

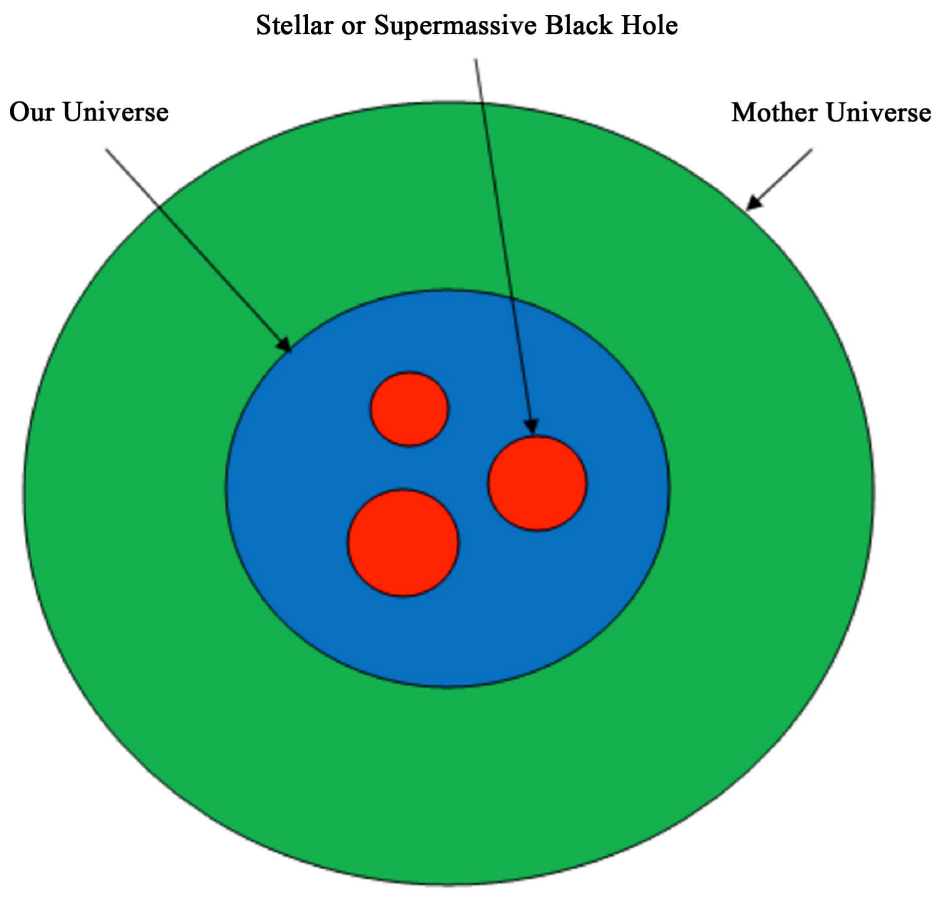

Figure 6. The innermost three layers of the entire space that is structured hierarchically up to mother universe (coded as green) [4] [5]. Here within our black hole universe (coded as blue), we only drew three child universes (coded as red), corresponding to the star-like or supermassive black holes. 
To see the multilayer structure of the space in a larger (or more complicate) view, we plot, in Figure 7, the innermost four layers of the black hole universe [4] [5]. It includes the grandmother universe, aunt universes, mother universe, sister universes, cousin universes, our universe itself, child universes, and niece universes. Here again for the sketch to be simple, we only drew a few of universes for each layer. If the whole space is finite, then the matter in the whole space is finite and thus the number of layers is finite. Otherwise, it has infinite layers and the outermost layer corresponds to the limit of zero degree for the absolute temperature, zero for the density, and infinity for the radius and mass. These four generations of the universe family can also be represented by a universe family tree (see Figure 8) [4] [5]. The mother and aunt universes are children of the grandmother universe. The cousin universes are children of the aunt universes. Our universe, the sister universes, and the cousin universes have their own children, which are the star-like or supermassive black holes.

The evolution of the space structure is iterative [4] [5]. In each of iterations, the matter reconfigures and the universe is renewed rather than a simple repeat or bouncing back. Figure 9 shows a series of sketches for the cartoon of the universe evolution in a single iteration from the present universe to the next similar one (from the top left to bottom right). This whole spacetime evolution process does not have the end and the beginning. As our universe expands, the child

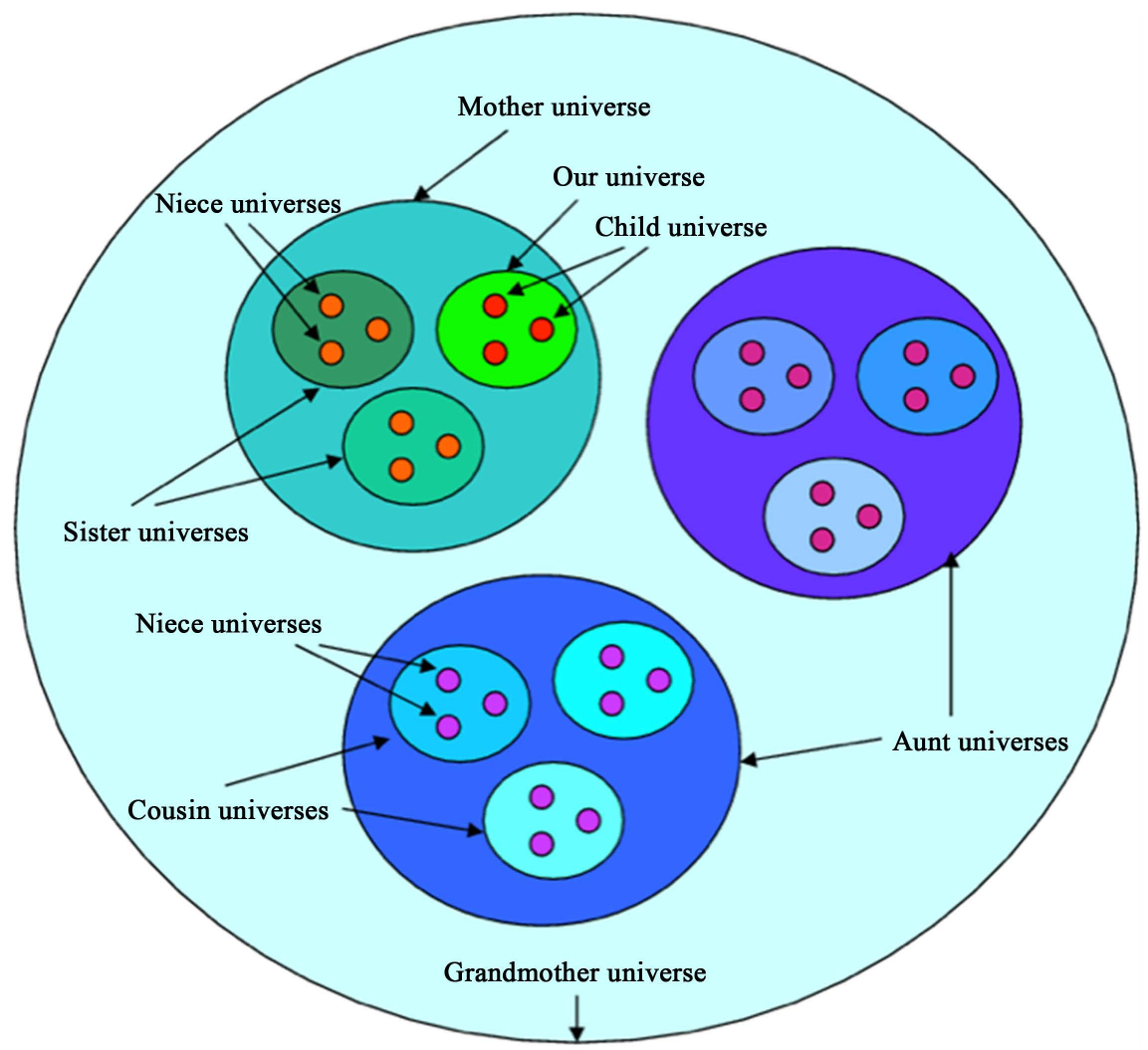

Figure 7. The innermost four layers of the entire space that is structured hierarchically up to grandmother universe [4] [5]. Here we also drew two sister universes that are parallel to our black hole universe and two aunt universes that are parallel to our mother universe. 


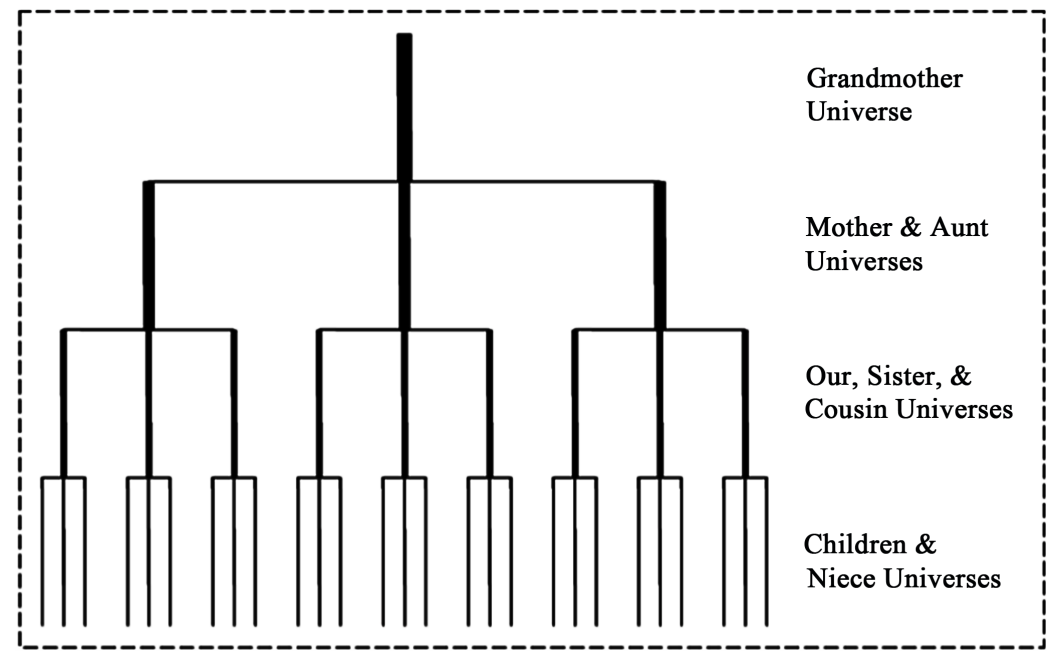

Figure 8. A family tree for the youngest four generations of the universe family [4] [5]. The generation one includes the child and niece universes; the generation two includes our universe itself and the sister universes; the generation three includes the mother and aunt universes; and the generation four includes the grandmother universe.
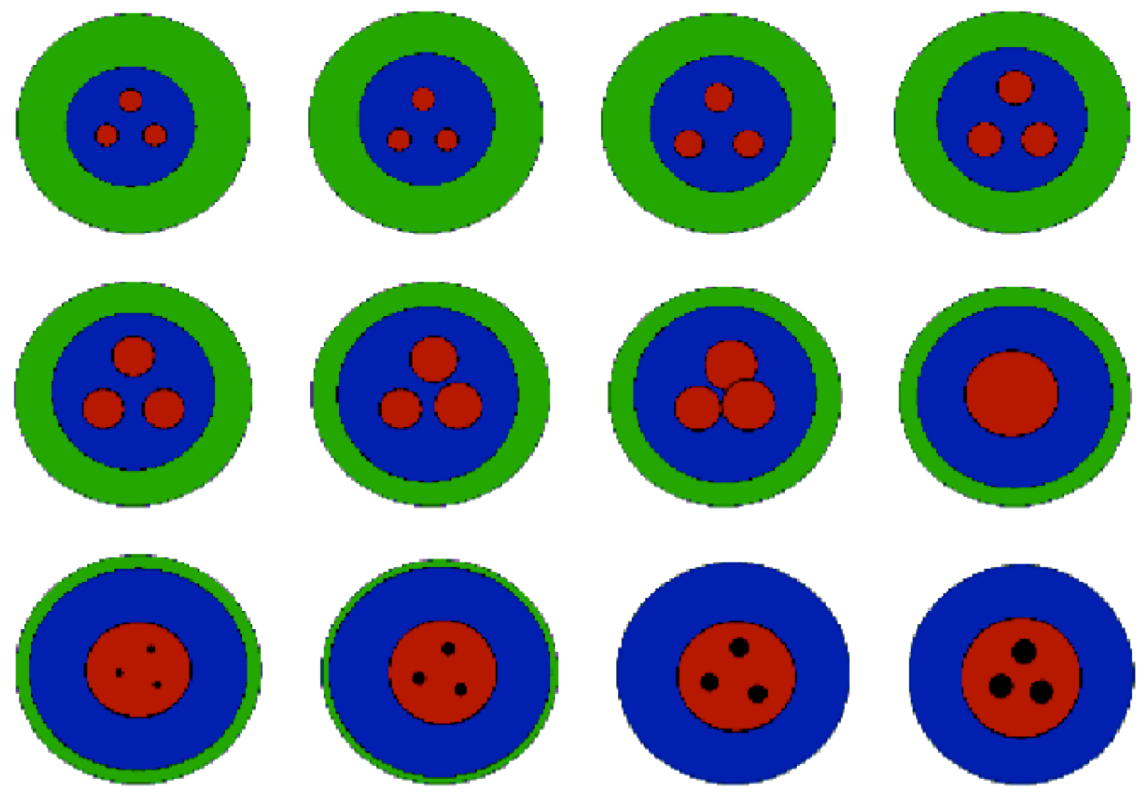

Figure 9. A series of sketches (or a cartoon, from left to right and then top row to bottom row) for the black hole universe to evolve in a single iteration from the present universe to the next similar one [4] [5]. This is an irreversible process, in which matter and spacetime reconfigure rather than a simple repeat or bouncing back. One universe is expanded to die out and a new universe is born from inside.

universes (i.e. the inside star-like and supermassive black holes) grow and merge each other into a new universe. Therefore, when one universe expands out, a new similar universe is born from inside. Like the living things in the nature, the universe passes through its own birth, growth, and death process and iterates this process endlessly. Its structure evolves iteratively forever without the beginning and end. 
There is nothing more natural than to consider the entire space to be infinite large without an edge and have infinite number of layers [4] [5]. For the outermost layer, the radius tends to infinity, while the density and absolute temperature both tend to zero. We call this outermost layer as the whole/grand space universe because it contains all universes. To represent this infinite layer structure of the whole space, we use the mathematical set concept (see Figure 10). We let the whole/grand space universe be the set (denoted by $U$ ) of all universes; the child universes (also the niece universes) are null sets $(C=\{\}$ or $N=\{\})$; our universe is a set of the child universes $(O=\{C, C, C, \cdots, C\})$; the sister universes are sets of the niece universes $(S=\{N, N, N, \cdots, N\})$; the mother universe is a set of our universe and the sister universes $(M=\{S, S, S, \cdots, O\})$; the aunt universes are sets of the cousin universes $(A=\{K, K, K, \cdots, K\})$; the grandmother universe is a set of the aunt universes and the mother universe $(G=\{A, A, A, \cdots, M\})$; and so on.

The black hole universe model gives a fantastic structure of the whole space. All universes are self-similar and governed by the same physics, the Einsteinian general theory of relativity with the FLRW metric, Mach-Schwarzschild $M-R$ relation, and positive curvature constant. The expansion of a black hole universe increases its mass and radius and decreases its density and temperature, but it does not alter the laws of physics. This infinite hierarchically layered space can also be represented as Figure 11. The top layer is the entire space, i.e. the grand universe. The bottom layer is a child universe. The second layer from the bottom is our universe. A child universe is a subspacetime of our universe; our universe is a subspacetime of the mother universe; the mother universe is a subspacetime of the grandmother universe; and so on. The total number of universes in the whole space is given by $n=\sum_{i=1}^{L} n_{i}$, where the subscript $i$ is the layer number,

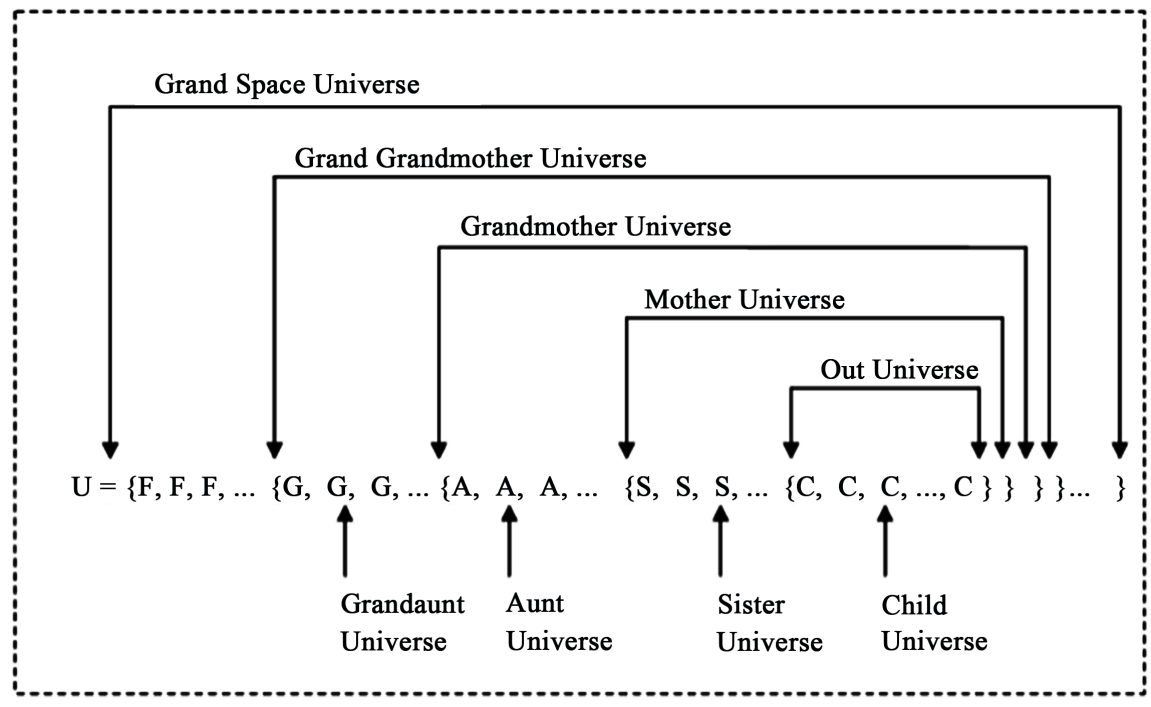

Figure 10. A mathematical representation of sets of universes for an infinite large and layered space [4] [5]. An inner layer universe set is a subset of the outer layer universe set. The niece and child universes are null sets because they do not contain any sub-spacetime. 


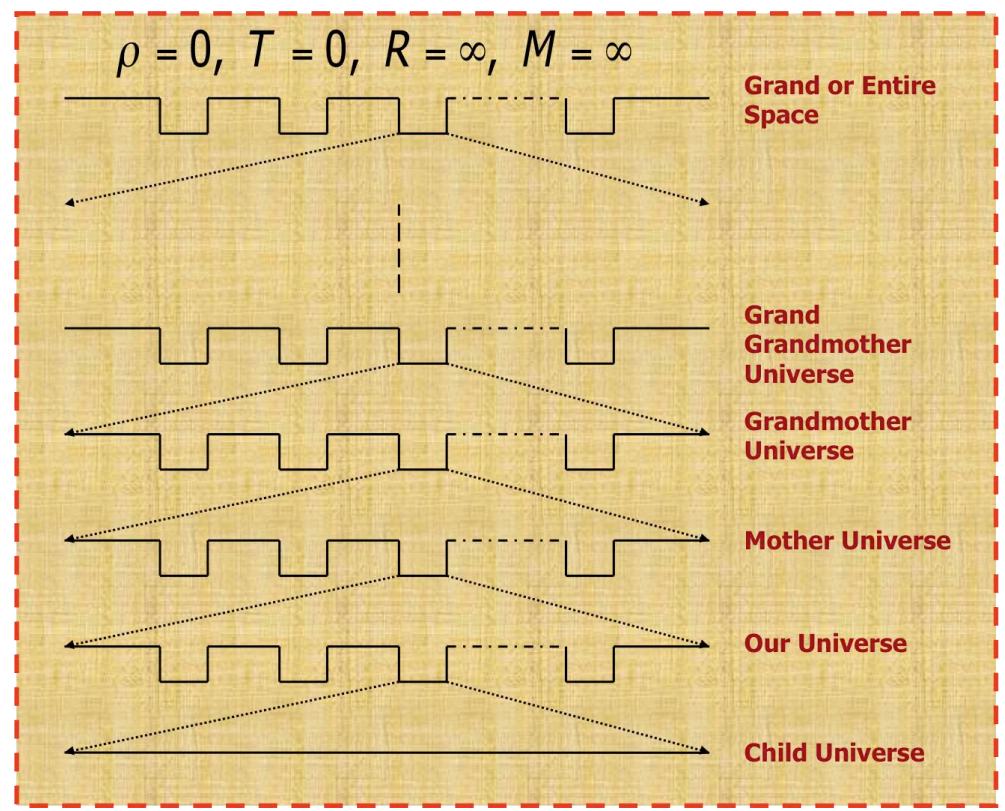

Figure 11. The entire space with infinite layers or subspacetimes [9] [18]. The bottom layer is a child universe or an empty spacetime. The child universe is a subspacetime of the universe in which we live. Similarly, our universe is a subspacetime of the mother universe, and so on. The top layer is the entire space of all subspacetimes.

$n_{i}$ is the number of universes in the $i^{\text {th }}$ layer, and $L$ refers to the number of layers in the whole space. For the four layer (or generation) black hole universes sketched in Figure 7 or Figure 8, we have $L=4$ and $n=27+9+3+1=40$. If the whole space includes infinite number of layers (i.e. $L=\infty$ ) and then the total number of universes is infinity (i.e. $n=\infty)$. To explain the observation of cosmic microwave background radiation, the black hole model of the universe also needs the entire space to have infinite layers [9] [18]. The map of cosmic background radiation measured by the Planck Satellite has provided the first hard evidence for multiverse. The black hole universe model is a multiverse model and thus supported by the recent Planck CMB measurements.

\subsection{The Law of Spacetime Acceleration}

When a spacetime or black hole including our black hole universe accretes matter in an increasing rate, i.e. $\ddot{M}(t)>0$, it accelerates its expansion [13] [21]. This regularity of spacetime is called the law of spacetime acceleration. It is combined with the law of spacetime expansion as the $2^{\text {nd }}$ law of black hole universe. The dimensionless deceleration parameter is usually defined as

$$
q(t) \equiv-\frac{R(t) \ddot{R}(t)}{\dot{R}^{2}(t)}=-\frac{M(t) \ddot{M}(t)}{\dot{M}^{2}(t)}
$$

where the double dot sign refers to the second order derivative with respect to time. From Equation (13), we see that whether the black hole universe accelerates or not depends on whether the double dot of mass is positive or not. For a positive $\ddot{M}(t)>0$, we have a negative deceleration parameter $q(t)<0$, i.e. ac- 
celeration of the universe.

According to the type Ia supernova measurements and their empirical distance-redshift relation, Daly et al. [50] obtained the deceleration parameter of the present universe as $q_{0} \sim-0.48$ for the flat FLRW metric of spacetime with $k=0$ (note that $q_{0}$ is smaller for $k=1$ ). To explain the recent acceleration of the universe, the big bang universe model suggests that the universe is dominated by dark energy up to about $75 \%$. In the black hole universe model, however, the universe accelerates because it inhales the outside matter in an increasing rate, i.e. a positive $\ddot{M}(t)>0$. To accelerate its expansion with $q_{0} \sim-0.48$, the present black hole universe only needs to inhale the outside matter in an increasing rate given by $\ddot{M}\left(t_{0}\right)=-q_{0} M_{0} H_{0}^{2}=-c^{2} q_{0} R_{0} H_{0}^{2} /(2 G) \sim 2.2 \times 10^{17} \mathrm{~kg} / \mathrm{s}^{2}$ (or about 110 solar masses per year square).

To explain the type Ia supernova measurements in accordance with the black hole universe model, we first solve Equation (13) to find the mass and Hubble parameter as functions of time. For a constant acceleration expansion universe, the time-dependent mass can be analytically solved from Equation (13) as,

$$
M(t)=M_{0}\left[(q+1) H_{0} t+1\right]^{\frac{1}{q+1}}
$$

from which the Hubble parameter is derived as

$$
H(t)=\frac{\dot{M}(t)}{M(t)}=\frac{H_{0}}{(q+1) H_{0} t+1}
$$

The time $t$ can be replaced by the redshift $z$, because

$$
1+z \equiv \frac{R_{0}}{R(t)}=\frac{M_{0}}{M(t)}=\left[(q+1) H_{0} t+1\right]^{-\frac{1}{q+1}}
$$

This mass-redshift relation, $M(t)=M_{0} /(1+z)$, does not depend on the deceleration parameter $q$. Using Equation (16) to replace the time of Equation (15), we obtain

$$
H(z)=H_{0}(1+z)^{q+1}
$$

To explain the type Ia supernova measurements according to the black hole universe model, we then determine the luminosity distance-redshift relation from the mass-time relation Equation (14) as

$$
d_{L}=(1+z) R_{0} \sin \left[\int_{t}^{0} \frac{c \mathrm{~d} t}{R(t)}\right]=(1+z) R_{0} \sin \left[\int_{t}^{0} \frac{c^{3} \mathrm{~d} t}{2 G M(t)}\right]
$$

Substituting Equation (14) into Equation (18), completing the integration and then using Equation (16), we have

$$
d_{L}=(1+z) R_{0} \sin \left[\frac{c^{3}}{2 G M_{0} H_{0}} \frac{1-(1+z)^{-q}}{q}\right]
$$

This redshift and luminosity distance relationship (Equation (19)) reduces to the Hubble law $z=d_{L} H_{0} / c$ at $z \ll 1$. 
Figure 12 plots the luminosity distance-redshift relation (red line) along with the type Ia supernova measurements (blue dots. Credit: Union 2.1 compilation of 580 SNIA data from Supernova Cosmology Project [51] [52]). In this plot the Hubble constant is chosen to be $H_{0}=70 \mathrm{~km} / \mathrm{s} / \mathrm{Mpc}$ and the deceleration parameter is chosen to be $q_{0}=-0.6$. In the upper panel of Figure 12, the distance modulus, which is defined by $\mu=5 \log _{10} d_{L}-5$ with $d_{L}$ in parsecs, is plotted as a function of redshift; while in the lower panel of Figure 12, the distance modulus difference between the measured SNIA data and analytical results derived from Equation (19). The results have shown that the black hole universe model can perfectly explain the measurements of type Ia supernovae.

Recently, the author pointed out that the luminosity distance-redshift relation that was usually applied to analyze the measurement of distant type Ia supernovae is an approximate expression that is only valid for nearby objects with
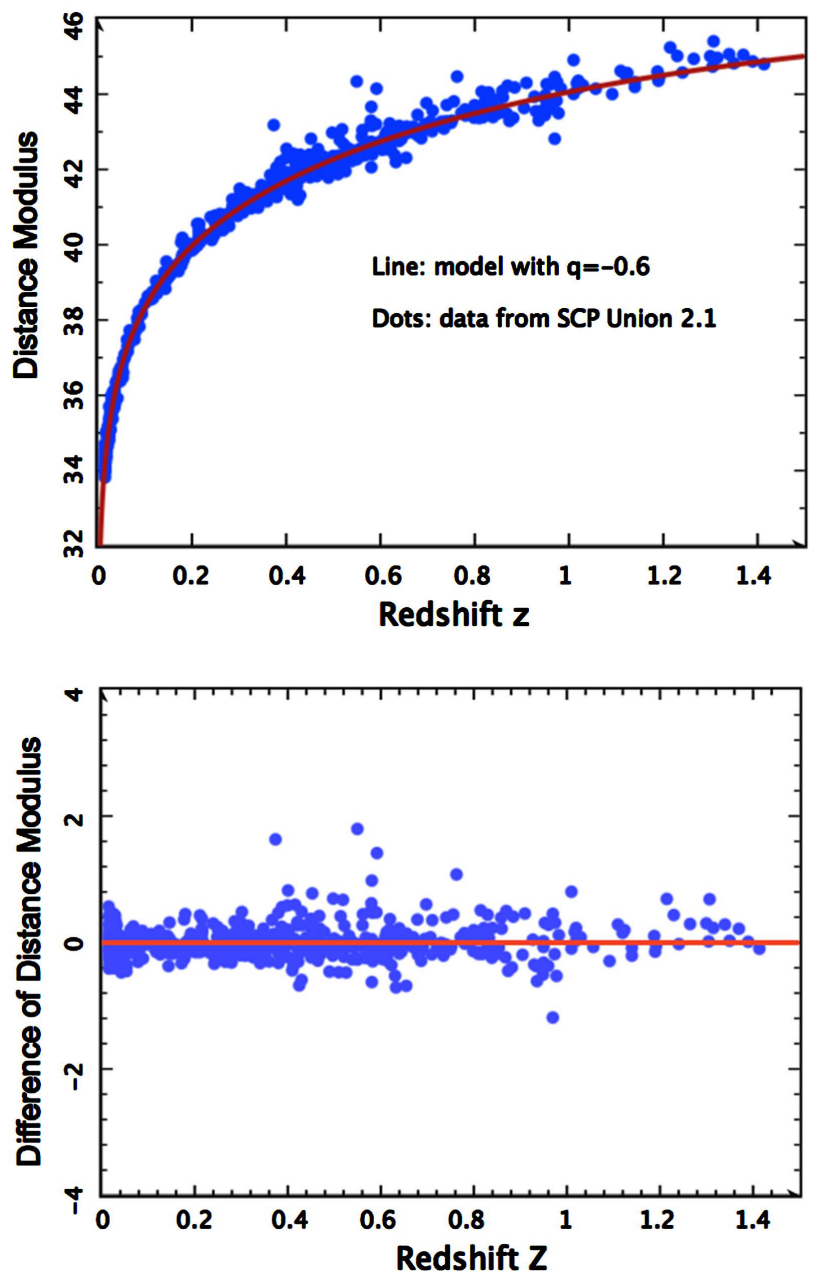

Figure 12. Luminosity distance-redshift relation of type Ia supernovae [13] [21]. Blue dots are measurements credited by the Union2 compilation of 580 SNeIa data from Supernova Cosmology Project [51] [52]. Red lines are analytical results from this study with $q=-0.6$. The upper panel plots the distance modulus as a function of redshift, while the lower panel plots the distance modulus difference between the measurement data and theoretical results. 
$z \ll 1$ [14] [15] [20]. The luminosity distances of all distant type Ia supernovae with $z \geq 1$ had been underestimated. Zhang [14] [20] has corrected the luminosity distance expression with an extra redshift factor $\sqrt{1+z}$, with which the type Ia supernova measurements do not lead to the acceleration of the universe and thus remove the need of dark energy.

In the black hole universe model, if we include this redshift factor, the luminosity distance expression (Equation (19)) becomes,

$$
d_{L}=(1+z)^{3 / 2} R_{0} \sin \left[\frac{c^{3}}{2 G M_{0} H_{0}} \frac{1-(1+z)^{-q}}{q}\right]
$$

Using this equation, we can also nicely fit the data of type Ia supernovae if the deceleration parameter is chosen about $q=0.5$ (see Figure 13). This corrected luminosity distance-redshift relation excludes the universe acceleration, so that removes the need of dark energy for the big bang universe model and the need of increasing its mass in an increasing rate for the black hole universe model.

\subsection{The Law of Spacetime Radiation Conservation}

According to Planck's law, the spectral energy density of the blackbody radiation within a black hole including the black hole universe can be written as

$$
u(v, T)=\frac{8 \pi h v^{3}}{c^{3}} \frac{1}{\exp \left(\frac{h v}{k_{B} T}\right)-1}
$$

where $v$ is the radiation frequency, $T$ is the temperature, $h$ is the Planck constant, and $k_{B}$ is the Boltzmann constant. In the SI unit system, the unit of $u(v, T)$ is $\mathrm{J} / \mathrm{m}^{3} / \mathrm{Hz}$. Figure 15 plots the spectral energy density as a function of frequency at temperature equal to $2.725,10^{4}, 10^{8}$, and $10^{12} \mathrm{~K}$, respectively [18].

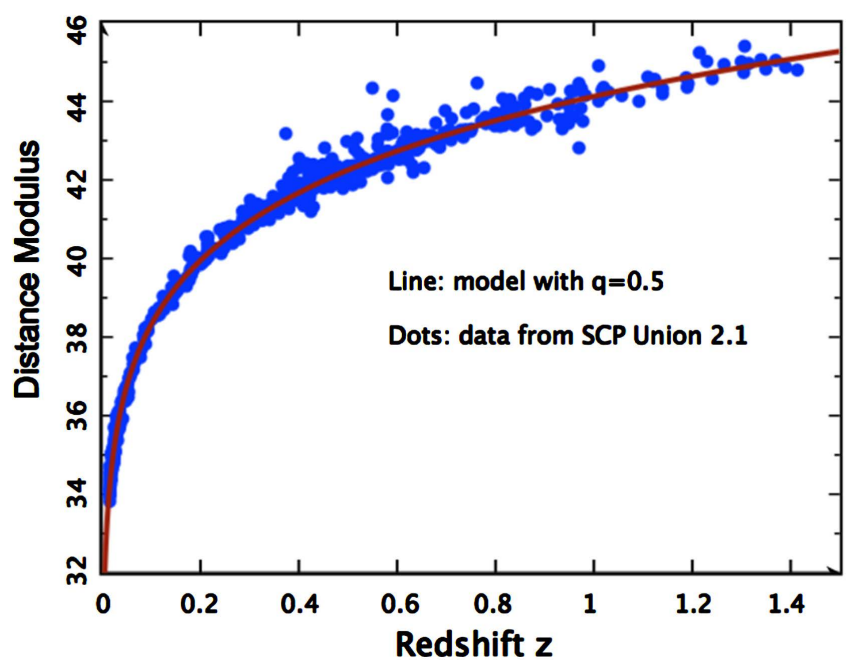

Figure 13. Distance modulus versus redshift, obtained from the corrected luminosity distance-redshift relation with the redshift factor $\sqrt{1+z}$ and $q=0.5$ (red line) [14] [20]. Blue dots are again the measurements credited by the Union2 compilation of 580 SNeIa data from Supernova. 
Integrating the spectral energy density (Equation (21)) with respect to the frequency of radiation in the entire range, we have the energy density of the blackbody radiation inside a black hole including the black hole universe,

$$
\rho_{\gamma} \equiv \frac{U}{V}=\frac{8 \pi^{5} k_{B}^{4}}{15 h^{3} c^{3}} T^{4}=\beta T^{4}
$$

where $U$ is the total radiation energy inside the black hole, $V$ is the volume of the black hole, and the constant $\beta$ is given by $\beta \equiv 8 \pi^{5} k_{B}^{4} /\left(15 h^{3} c^{3}\right) \sim 7.54 \times 10^{-16}$ $\mathrm{J} / \mathrm{m}^{3} / \mathrm{K}^{4}$. Inside a black hole with the four temperatures given in Figure 15 , the energy densities of radiation are $\sim 4.15 \times 10^{-14}, 7.54,7.54 \times 10^{16}, 7.54 \times 10^{32} \mathrm{~J} / \mathrm{m}^{3}$, respectively.

As a spacetime or black hole including the black hole universe accretes its outside matter and radiation, it expands its volume from $V$ to $V+\mathrm{d} V$ or radius from $R$ to $R+\mathrm{d} R$. This type of expansion does not geometrically stretch the space of itself, instead it just takes the space as well as the matter and radiation from the outside. Considering that the increase of the Planck radiation energy within the black hole equals to the radiation energy inhaled from the outside space, we have

$$
\beta(T+\mathrm{d} T)^{4}(V+\mathrm{d} V)-\beta T^{4} V=\beta T_{p}^{4} \mathrm{~d} V
$$

where $T$ is the temperature of the black hole, $\mathrm{d} T$ is the change (or decrease because it is negative) of the temperature due to the expansion of the black hole, and $T_{p}$ is the temperature outside the black hole, i.e. the temperature of the mother black hole or universe. Since $\mathrm{d} V / V=3 \mathrm{~d} R / R$, we can reform Equation (23) by a first order development as

$$
\frac{\mathrm{d} T}{\mathrm{~d} R}=-\frac{3 T}{4 R}\left[1-\left(\frac{T_{p}}{T}\right)^{4}\right]
$$

This equation governs the thermal history of the black hole universe that grew up from a star-like black hole through a supermassive black hole and a mini universe to the present state [9] [18]. The solution of Equation (24) for the dependence of $T$ on $R$ depends on $T_{p}$ or on the relation between $T$ and $T_{p}$. In the early studies cited above, Equation (24) was solved for the present black hole universe that grew up from a hot star-like black hole through a supermassive black hole.

For hot star-like or supermassive black holes, the temperatures inside should be much greater than the temperatures outside, i.e. $T \gg T_{p}$. In this case Equation (24) approximately reduces to

$$
\frac{\mathrm{d} T}{\mathrm{~d} R}=-\frac{3 T}{4 R}
$$

which can be simply solved as

$$
R^{3} T^{4}=C
$$

where $C$ is an integral constant. Therefore, the temperature of a star-like or supermassive black hole decreases as it expands in size according to $T \propto R^{-3 / 4}$. 
Substituting Equation (26) into Equation (22), we obtain the total radiation energy inside a star-like or supermassive black hole to be a constant and thus independent of its size,

$$
U=V \beta T^{4}=\frac{4}{3} \pi \beta R^{3} T^{4}=\frac{4}{3} \pi \beta C=\text { Constant }
$$

This interesting result indicates that the blackbody radiation energy inside a star-like or supermassive black hole remains the same no matter it is static or dynamic. Attaining matter and growing its size do not increase its total radiation energy. Here, we call this regularity of constant radiation energy as the law of radiation energy conservation (or the $3^{\text {rd }}$ law of black hole universe).

Figure 14 has shown that the spectral energy density of blackbody radiation inside a spacetime or black hole rapidly increases with the temperature and frequency. Inside a star-like black hole with temperature of $10^{12} \mathrm{~K}$, the blackbody radiation dominates at the frequency of gamma rays. The radiation maximizes its spectral energy density up to $\sim 10^{25} \mathrm{~J} / \mathrm{m}^{3} / \mathrm{Hz}$ at frequency of $\sim 10^{23} \mathrm{~Hz}$. Inside a massive or supermassive black hole with temperature of $10^{8} \mathrm{~K}$, the blackbody radiation dominates at the frequency of $\mathrm{X}$-rays. The radiation maximizes its spectral energy density up to $\sim 10^{12} \mathrm{~J} / \mathrm{m}^{3} / \mathrm{Hz}$ at frequency of $\sim 10^{19} \mathrm{~Hz}$.

A black hole, when it accretes its ambient matter or merges with another black hole, becomes dynamic. A dynamic black hole has a broken event horizon and thus cannot hold the inside hot (or high-frequency) blackbody radiation, which flows or leaks out of it and produces X-ray flares or gamma ray bursts. Dynamic star-like black holes with thousand billions of Kelvins (i.e. $\sim 10^{12} \mathrm{~K}$ ) radiate gamma rays, while dynamic massive or supermassive black holes with hundred millions of Kelvins (i.e. $\sim 10^{8} \mathrm{~K}$ ) radiate $\mathrm{X}$-rays such as $\mathrm{X}$-ray emissions from

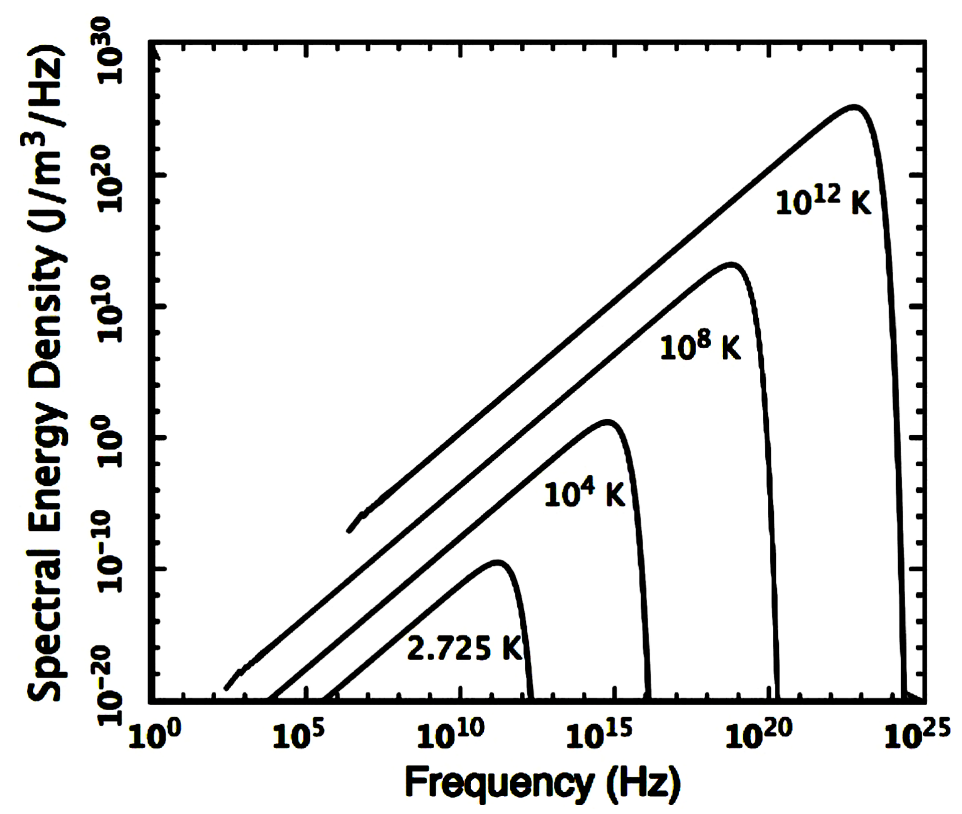

Figure 14. The spectral energy density of blackbody radiation as a function of radiation frequency at temperature equal to $2.725,10^{4}, 10^{8}$, and $10^{12} \mathrm{~K}$, respectively. 
quasars, supermasive black holes with billions of solar masses, and X-ray flares from Sagitarius $\mathrm{A}^{\star}$, a massive black hole with millions of solar masses at the Milky Way center. This section describes how these dynamic properties of black holes are explained according to the black hole model of the universe [12] [16] [19] [22] [23] [24].

To reveal the possible thermal history of the black hole universe, we have considered a general case that the black hole universe decreases its relative temperature in a rate slightly greater than the mother universe as [18]

$$
\frac{\mathrm{d} T_{p}}{T_{p}}=q \frac{\mathrm{d} T}{T}
$$

which is equivalent to

$$
T_{p}=\alpha T^{q} \text { or } \frac{T_{p}}{T}=\alpha T^{-\delta}
$$

Here $q$ is a constant slightly less than $1 ; \delta \equiv 1-q$ is a small number; and $\alpha$ can be derived from $q$ or $\delta$ according to the temperature and radius of the present universe. The other cases such as $q=1$ or $q$ to be significantly smaller than the unity could not explain the measurement of cosmic microwave background radiation as shown in [18].

In this case with $q$ slightly smaller than the unity, the solution of Equation (24) can be analytically obtained as

$$
T=R^{-3 / 4}\left(\alpha^{4} R^{3 \delta}+T_{s}^{4 \delta} R_{s}^{3 \delta}\right)^{1 / 4 \delta}
$$

where the constant $\alpha$ is given by

$$
\alpha=\left[T_{0}^{4 \delta}-\left(\frac{R_{s}}{R_{0}}\right)^{3 \delta}\right]^{1 / 4}
$$

Choosing $q$ or $\delta$ appropriately, we can completely determine the thermal history of the black hole universe that evolved from a hot star-like black hole with temperature $T_{s}$ and radius $R_{s}$ to the present universe with temperature $T_{0}$ and radius $R_{0}$. In Figure 15, the radiation temperature of the black hole universe is plotted as a function of the universe radius with a fixed $q=0.93$ or $\delta=0.07$. Here we have chosen $T_{0}=2.725 \mathrm{~K}, R_{0}=13.7$ billion light years, $R_{s}=9 \mathrm{~km}, T_{s}=10^{12} \mathrm{~K}$ (solid line), $T_{s}=10^{11} \mathrm{~K}$ (dotted-dashed line), and $T_{s}=4 \times 10^{12} \mathrm{~K}$ (dashed line).

It is seen that all temperature lines are concave downward and approach $\sim 2.725 \mathrm{~K}$ as the black hole universe expands to the present size. Therefore, the initial temperature of the star-like black hole $T_{s}$ is not critical to the present universe. The reason is because most matter and radiation are from the mother universe. This reason also explains why all other physical properties of the star-like black hole, including its size (or mass), angular momentum, and charge, and the evolution of the early universe are not critical to the present universe. Furthermore, the early process of material accretion and black hole mergers do not have significant leftover in the present universe. 


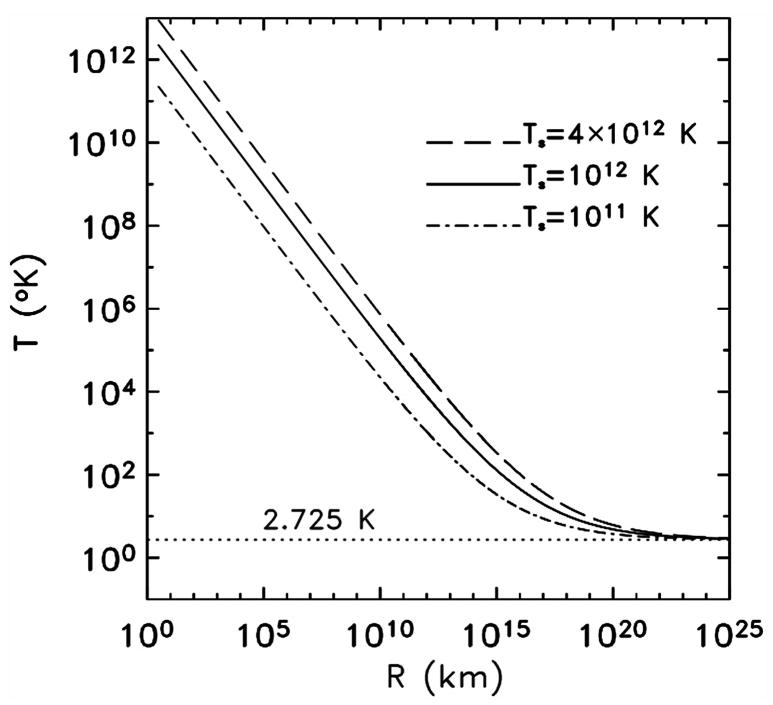

Figure 15. The possible thermal history of the black hole universe [18]. The solid line corresponds to $T_{s}=10^{12} \mathrm{~K}$, the dotted-dashed line corresponds to $T_{s}=10^{11} \mathrm{~K}$, and the dashed line corresponds to $T_{s}=4 \times 10^{12} \mathrm{~K}$. All temperature lines are curved by concaving downward and approach $\sim 2.725 \mathrm{~K}$ at the present as the black hole universe expands to the present size.

\subsection{The Law of Spacetime Entropy Increase}

A spacetime or black hole including our black hole universe expands when it accretes matter and radiation from its outside. The expansion also alters its entropy given by [53]

$$
\mathrm{d} S \equiv \frac{\mathrm{d} Q}{T}=\frac{c^{2}}{T} \mathrm{~d} M+\frac{\beta T_{b}^{4}}{T} \mathrm{~d} V=\left(\frac{c^{4}}{2 G T}+\frac{4 \pi \beta T_{b}^{4} R^{2}}{T}\right) \mathrm{d} R
$$

The first term of Equation (32) is the entropy increase due to the accretion of matter and the second term of Equation (32) is the entropy increase due to the accretion of radiation. It is seen from Equation (32) that the entropy of a spactime or black hole including our black hole universe does not decrease, $\mathrm{d} S \geq 0$, because $\mathrm{d} R \geq 0$. This regularity is called the law of spactime entropy increase (or the $4^{\text {th }}$ law of black hole universe). Figure 16 plots $\mathrm{d} S / \mathrm{d} R$ as a function of $R$. To plot this, we have used the temperature-radius (T-R) relations and constants associated, Equations (30) and (31).

Integrating Equation (32), we have the entropy of a spacetime or black hole with radius $R$ or mass $M=c^{2} R /(2 G)$,

$$
S-S_{s}=\int_{R_{s}}^{R}\left(\frac{c^{4}}{2 G T}+\frac{4 \pi \beta T_{b}^{4} R^{2}}{T}\right) \mathrm{d} R
$$

where $S_{s}$ is the entropy of the reference black hole with mass $M_{s}=3$ solar masses (or radius $R_{s}=8.89 \mathrm{~km}$ ) and temperature $T_{s}=10^{12} \mathrm{~K}$. Using Equations (30) and (31) to relate the temperatures $T_{b}$ and $T$ to the radius $R$, we can numerically solve Equation (33) and find the entropy of a spacetime or black hole as a function of its radius or mass. 


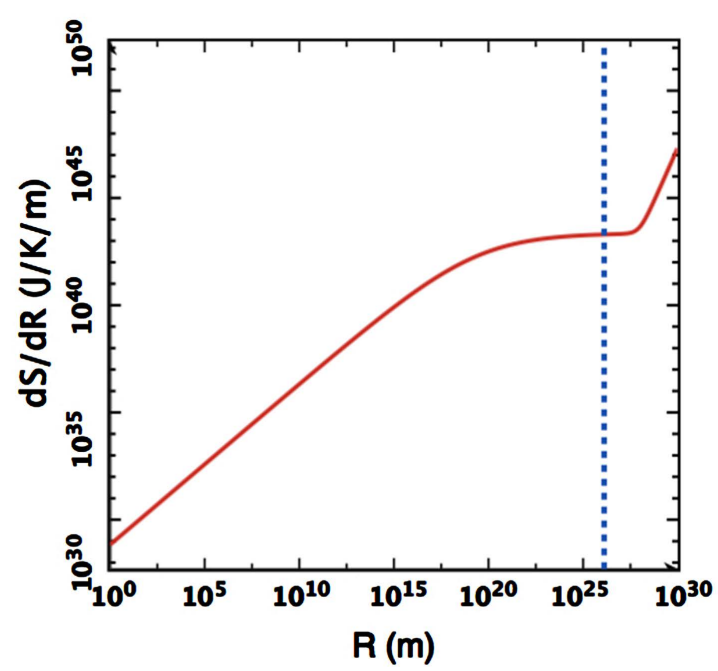

Figure 16. The slop for the increase of entropy of a spacetime or black hole including our black hole universe is plotted as a function of the radius.

For a star-like or supermassive black hole, we have $T_{p} \ll T$ and $T=T_{s}\left(R_{s} / R\right)^{3 / 4}$ as seen from Equation (26). Further using the Mach-Schwarzschild M-R relation Equation (1), we have $T=T_{s}\left[R_{s} c^{2} /(2 G M)\right]^{3 / 4}$. Then, the entropy of a star-like or supermassive black hole can be obtained by the following,

$$
S_{B H}=\int_{0}^{M} \frac{c^{2}}{T} \mathrm{~d} M=\frac{4 c^{2}}{7 T_{s}}\left(\frac{2 G}{R_{s} c^{2}}\right)^{3 / 4} M^{7 / 4}
$$

It is seen that the entropy of a star-like or supermassive black hole is proportional to the mass or radius according to $S_{B H} \propto M^{7 / 4}$ or $S_{B H} \propto R^{7 / 4}$. Figure 17 plots the entropy of a star-like or supermassive black hole as a function of the black hole mass (solid red line). Let the mass equal one solar mass $M=M_{\text {sun }}$, then, from Equation (34), we obtain the entropy of 1-solar mass black hole as $S_{\text {sun }}=3 \times 10^{35} \mathrm{~J} / \mathrm{K}$. For a supermassive black hole with one billion solar masses, its entropy is equal to about $10^{50} \mathrm{~J} / \mathrm{K}$. In Figure 18, we also plot the entropy of a star, $S_{\text {star }}$, which is usually estimated according to the Sackur-Tetrode equation, as a function of its mass,

$$
S_{\text {star }}=k_{B} N_{p}\left\{\ln \left[\frac{V_{\text {star }}}{N_{p}}\left(\frac{2 \pi k_{B} m_{p} T_{\text {star }}}{h^{2}}\right)^{3 / 2}\right]+\frac{5}{2}\right\} \sim 18 k_{B} N_{p}
$$

where $N_{p}$ is the number density of proton in the star, $m_{p}$ is the mass of proton, $V_{\text {star }}$ is the volume of the star.

From Figure 17, it is seen that the entropy of a black hole with mass less than about 10 solar masses is lower than that of a star with the same mass. This implies that a star with mass less than 10 solar masses cannot directly produce or collapse into a black hole with the same mass at the end of its life. This is in agreement with Enistein's general theory of relativity, which suggests, to produce a star-like black hole, that the parent star must be 20 or more massive than the Sun. As a typical example, considering that a star with 20 solar masses forms a 


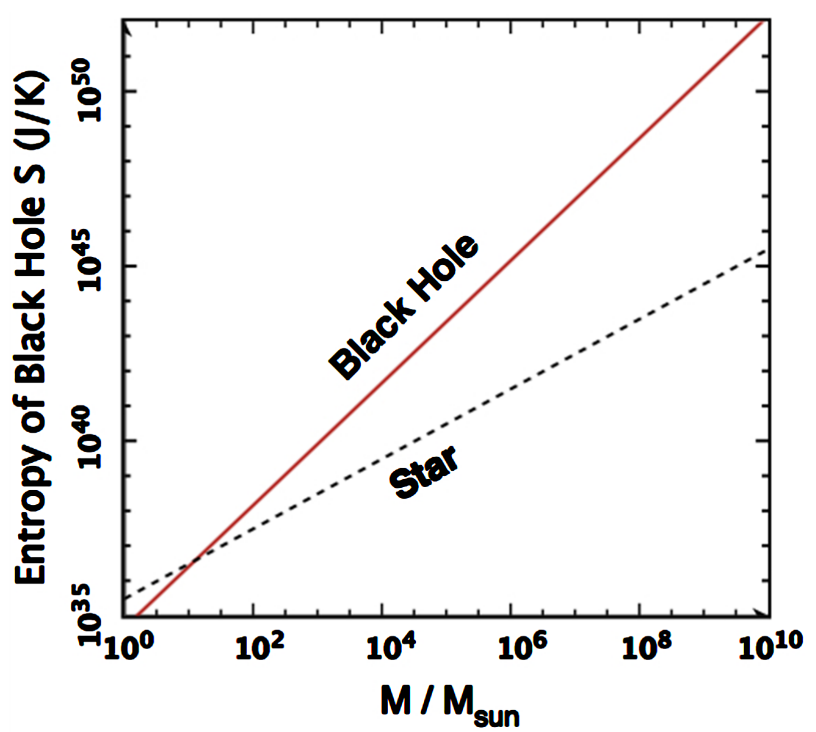

Figure 17. The entropies of a black hole and a star are plotted as functions of the mass. Results indicate that the entropy of black hole increases with mass faster than that of star does. But, the entropy of a black hole with the mass if less than 10 solar masses is lower than that of a star with the same mass.

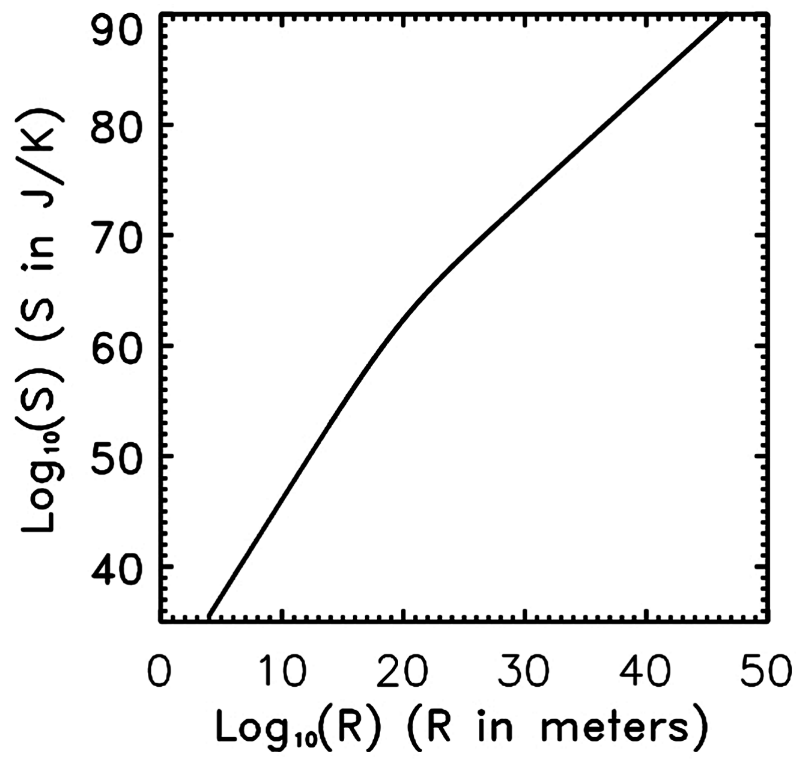

Figure 18. Entropy of a spacetime or black hole including our black hole universe. It is ploted as a function of the radius in logarithm scales. As starlike black hole grows up into a universe as big as the present universe, its entropy increases from about $10^{35} \mathrm{~J} / \mathrm{K}$ to about $10^{70} \mathrm{~J} / \mathrm{K}$.

black hole with the same mass, the entropy increases to $10^{37} \mathrm{~J} / \mathrm{K}$ from $10^{36} \mathrm{~J} / \mathrm{K}$. If the black hole formed has only 5 solar masses by a supernova explosion, the entropy decreases to $10^{35} \mathrm{~J} / \mathrm{K}$ from $10^{36} \mathrm{~J} / \mathrm{K}$ because the supernova explosion has brought a significant amount of entropy away. The entropy of a star with 50 solar masses has entropy of $\sim 10^{37} \mathrm{~J} / \mathrm{K}$. When it forms a black hole with 10 solar masses, which has entropy of $7 \times 10^{36} \mathrm{~J} / \mathrm{K}$, at the end of its life after supernova 
explosion, the supernova explosion carries about $3 \times 10^{36} \mathrm{~J} / \mathrm{K}$ or more entropy away.

Conventionally, the entropy of a black hole is calculated according to the Hawking radiation. Considering the quantum effect near the event horizon, Hawking [42] theoretically showed that a black hole, even though in the static state (no accretion and no merger), can radiate blackbody emission called Hawking radiation with temperature of $\sim 1.23 \times 10^{23} / \mathrm{M} \mathrm{K}$ and power of $\sim 3.56 \times$ $10^{32} / \mathrm{M}^{2} \mathrm{~W}$. Here $M$ is the mass of black hole in kilograms. For a star-like or more massive black hole, both the temperature and power of the Hawking radiation are negligible small. In accordance with the temperature of Hawking radiation, the entropy of the black hole is obtained as $3 \times 10^{-7} \mathrm{M}^{2} \mathrm{~J} / \mathrm{K}$, which is about $10^{20}$ times greater than that of star with the same mass as well as that of a dynamic black hole.

Now, we come back to Equation (33), to plot, in Figure 18, the numerically integrating result for the entropy of a black hole including our black hole universe as a function of radiation. It is seen that, for a star-like black hole to grow into a universe as big as the present universe, its entropy increases from about $10^{35} \mathrm{~J} / \mathrm{K}$ to about $10^{70} \mathrm{~J} / \mathrm{K}$. The entropy of the present highly ordered universe is still keeping quickly growing. Since the black hole universe is not an isolated system, its entropy is mostly coming from the mother universe due to accretions of matter and radiation. This increase does not lose the order significantly. This explains why the present universe, although it fully grows with temperature less than $3 \mathrm{~K}$, is still highly ordered.

\section{Discussions and Conclusions}

The black hole universe is not an isolated spacetime with $k=1$ [4] [5]. Its mass must increase when it expands because $M(t) / R(t)=c^{2} /(2 G)=$ Constant. The density decreases by inversely proportional to the square of the radius (or the mass) of the black hole universe. This mass-radius or density-radius relation excludes the flatness and horizon problems. Both black hole and big bang universes are governed by the same physics-Einstein's general relativity with the FLRW metric of spacetime. The big bang universe model suggests that the observable and non-observable spaces are isotropically uniform, while the black hole universe model considers them to be hierarchically layered. Big bang universe is an isolated system with $\rho(t) R^{3}(t)=$ Constant when matter is dominant and $k=0$, while black hole universe is a semi-open system with $\rho(t) R^{2}(t)=$ Constant and $k=1$. Mathematically, there is only a small change from the big bang universe model to the black hole universe model, but physically the two universe models lead to completely different results.

As a summary, the author has examined and overviewed thoroughly the new black hole universe model and completely described its development from the three fundamentals and its creative explanations to the existing observations of the universe. From this comprehensive investigation of this new cosmological model, the author has further revealed the fundamental regularities and laws of 
the black hole universe with respect to the spacetime origin, structure, evolution, equilibrium, expansion, acceleration, radiation energy, and entropy variation. These efforts will help us to uncover various regularities and mysteries of the universe. The results indicate that, standing on 1) the principle of spacetime black hole equivalence, 2) the cosmological principle of spacetime isotropy and homogeneity, and 3) the Einsteinian general theory of relativity, the black hole universe model can self-consistently describe the origin, structure, evolution, expansion, and acceleration of the universe, quantitatively explain the measurements of cosmic microwave background radiation, type Ia supernovae's luminosity distance and redshift, and dynamic properties of star-like, massive, and supermassive black holes such as gamma-ray bursts, X-rays flares from galactic centers, and quasars, and fully overcome the difficulties of the conventional model of the universe such as the problems of horizon, flatness, monopole, inflation, dark matter, dark energy, and so on.

The black hole universe is ruled by one theory of spacetime (i.e. the Einsteinian general theory of relativity), based on two principles of spacetime (i.e. the principle of spacetime black hole equivalence and the cosmological principle of spacetime isotropy and homogeneity), regulated with five laws of spacetime (i.e. the conversion of spactieme mass-radius ratio, the equilibrium of static spacetime, the expansion and acceleration of dynamic spacetime, the conservation of childish spacetime radiation energy, and the entropy increase of spacetime). It is governed by the Friedmann equation that can be derived from Einstein's general theory of relativity, with the FLRW metric that can be derived from the cosmological principle, the Mach-Schwarzschild mass-radius ratio, and the positive curvature of 3D space that can be obtained from the principle of spacetime black hole equivalence. Mathematically, the black hole universe is slightly different from the big bang universe (one has mass-radius ratio constant and $k=1$, while another has mass constant and $k=0$ ), but physically they are completely different.

\section{Acknowledgements}

The work that was done previously and involves partially with students was supported by the NSF/REU (Grant \#: PHY-1263253 and PHY-1559870) at Alabama A \& M University. The author is also thankful to the University's Title III Program for travel support of his attending conferences.

\section{Conflicts of Interest}

The authors declare no conflicts of interest regarding the publication of this paper.

\section{References}

[1] Brans, C.H. and Dicke, R. H. (1961) Physical Review, 124, 925-935. https://doi.org/10.1103/PhysRev.124.925

[2] Zhang, T.X. (2006) Astrophysical Journal Letters, 636, L61-L64. https://doi.org/10.1086/500255 
[3] Zhang, T.X. (2010) Astrophysical Journal Letters, 725, L117-L120. https://doi.org/10.1088/2041-8205/725/2/L117

[4] Zhang, T.X. (2007) American Astronomical Society $211^{\text {th }}$ Meeting.

[5] Zhang, T.X. (2009) Progress in Physics, 5, 3-11.

[6] Zhang, T.X. (2014) Observation and Theory for Multiverse. Special Session (The Oral Session \#304) of the 224 ${ }^{\text {th }}$ AAS Meeting, Boston, 7-11 June 2014.

[7] Zhang, T.X. (2014) American Astronomical Society $224^{\text {th }}$ Meeting.

[8] Zhang, T.X. (2009) American Astronomical Society $213^{\text {rd }}$ Meeting.

[9] Zhang, T.X. (2009) American Astronomical Society $214^{\text {th }}$ Meeting.

[10] Zhang, T.X. (2010) American Astronomical Society $215^{\text {th }}$ Meeting.

[11] Zhang, T.X. (2011) American Astronomical Society $217^{\text {th }}$ Meeting.

[12] Zhang, T.X. (2012) American Astronomical Society $219^{\text {th }}$ Meeting.

[13] Zhang, T.X. (2012) American Astronomical Society $220^{\text {th }}$ Meeting.

[14] Zhang, T.X. (2013) American Astronomical Society $221^{\text {st }}$ Meeting.

[15] Zhang, T.X. (2013) American Astronomical Society $222^{\text {nd }}$ Meeting.

[16] Zhang, T.X. (2014) American Astronomical Society $223^{\text {rd }}$ Meeting.

[17] Zhang, T.X. (2016) American Astronomical Society $228^{\text {th }}$ Meeting.

[18] Zhang, T.X. (2010) Astrophysics and Space Science, 330, 157-165. https://doi.org/10.1007/s10509-010-0372-4

[19] Zhang, T.X. (2013) Proceeding of GRB 2013 Symposium, SNSN-323-63.

[20] Zhang, T.X. (2013) Progress in Physics, 9, 33-38.

[21] Zhang, T.X. and Frederick, C. (2014) Astrophysics and Space Science, 349, 567-573. https://doi.org/10.1007/s10509-013-1644-6

[22] Zhang, T.X. (2015) Astrophysics and Space Science, 358. https://doi.org/10.1007/s10509-015-2409-1

[23] Zhang, T.X., Wilson, C. and Schamschula, M.P. (2016) Progress in Physics, 12, 61-67.

[24] Zhang, T.X., Naka, P. and Guggilla, P. (2016) 8th Huntsville Gamma-Ray Burst Symposium, Huntsville, 24-28 October 2016, LPI Contribution No. 1962, Article ID: 4028.

[25] Zhang, T.X. (2016) Progress in Physics, 12, 353-361.

[26] Arp, H., et al. (2004) An Open Letter to the Scientific Community. New Scientists.

[27] Guth, A.H. (1981) Physical Review D, 23, 347-356. https://doi.org/10.1103/PhysRevD.23.347

[28] Riess, A.G., et al. (1998) The Astronomical Journal, 116, 1009-1038. https://doi.org/10.1086/300499

[29] Perlmutter, S., et al. (1999) Astrophysical Journal, 517, 565-586. https://doi.org/10.1086/307221

[30] Schwarzschild, K. (1916) Mathematical Physics, 1916, 189-196.

[31] Sciama, D.W. (1953) Monthly Notices of the Royal Astronomical Society, 113, 34-42. https://doi.org/10.1093/mnras/113.1.34

[32] Davidson, M.W. (1957) Monthly Notices of the Royal Astronomical Society, 117, 212-224. https://doi.org/10.1093/mnras/117.2.212

[33] Slezak, M. (2014) Cosmic Inflation Is Dead, Long Live Cosmic Inflation. New 
Scientists, September 25.

[34] Cortes, M., Liddle, A.R. and Parkinson, D. (2014) Physical Review D, 92, Article ID: 063511. https://doi.org/10.1103/PhysRevD.92.063511

[35] Abbott, B.P., et al. (2016) Physical Review Letters, 116, Article ID: 061102. https://doi.org/10.1103/PhysRevLett.116.061102

[36] Friedmann, A. (1922) Zeitschrift fur Physik, 10, 377-386. https://doi.org/10.1007/BF01332580

[37] Lemaitre, G. (1931) Monthly Notices of the Royal Astronomical Society, 91, 483-490. https://doi.org/10.1093/mnras/91.5.483

[38] Robertson, H.P. (1935) Astrophysical Journal, 82, 284-301. https://doi.org/10.1086/143681

[39] Walker, A.G. (1937) Proceedings of the London Mathematical Society, 42, 90-127. https://doi.org/10.1112/plms/s2-42.1.90

[40] Einstein, A. (1916) Annalen der Physik, 354, 769-822. https://doi.org/10.1002/andp.19163540702

[41] Einstein, A. (1905) Annalen der Physik, 322, 891-921. https://doi.org/10.1002/andp.19053221004

[42] Hawking, S.W. (1974) Nature, 248, 30-31. https://doi.org/10.1038/248030a0

[43] Friedmann, A. (1924) Zeitschrift fur Physik, 21, 326-332. https://doi.org/10.1007/BF01328280

[44] Hubble, E. (1929) Proceedings of National Academy of Science of the United States of America, 15, 168-173. https://doi.org/10.1073/pnas.15.3.168

[45] Suyu, S.H., et al. (2010) Astrophysical Journal, 711, 201-221. https://doi.org/10.1088/0004-637X/711/1/201

[46] Tytler, D., et al. (2004) Astrophysical Journal, 617, 1-28. https://doi.org/10.1086/425226

[47] Einstein, A. (1917) Sitzungsberichte der Koniglich Preussbischen Akademie der Wissenschaften (Berlin), 8, 142-152.

[48] Zhang, T.X. (2018) Journal of Modern Physics, 9, 433-442.

[49] Shakura, N.I. and Syuanyaev, R.A. (1973) Astronomy and Astrophysics, 24, 337-355.

[50] Daly, R.A., Djorgovski, S.G., Freeman, K.A., Matthew, P., O’Dea, C.P., Kharb, P. and Baum, S. (2008) Astrophysical Journal, 677, 1-11. https://doi.org/10.1086/528837

[51] Amanullah, R., et al. (2010) The Astrophysical Journal, 716, 712-738. https://doi.org/10.1088/0004-637X/716/1/712

[52] Suzuki, N., et al. (2012) The Astrophysical Journal, 746, Article No. 85.

[53] Zhang, T.X. (2018) Entropy of Black Hole Universe, Astrophysics and Space Science. (To be Submitted) 